\title{
The formation of relativistic plasma structures and their potential role in the generation of cosmic ray electrons
}

\author{
M. E. Dieckmann \\ Department of Science and Technology (ITN), Linköping University, 60174 Norrköping, Sweden \\ Received: 20 February 2008 - Revised: 28 August 2008 - Accepted: 10 September 2008 - Published: 3 November 2008
}

\begin{abstract}
Recent particle-in-cell (PIC) simulation studies have addressed particle acceleration and magnetic field generation in relativistic astrophysical flows by plasma phase space structures. We discuss the astrophysical environments such as the jets of compact objects, and we give an overview of the global PIC simulations of shocks. These reveal several types of phase space structures, which are relevant for the energy dissipation. These structures are typically coupled in shocks, but we choose to consider them here in an isolated form. Three structures are reviewed. (1) Simulations of interpenetrating or colliding plasma clouds can trigger filamentation instabilities, while simulations of thermally anisotropic plasmas observe the Weibel instability. Both transform a spatially uniform plasma into current filaments. These filament structures cause the growth of the magnetic fields. (2) The development of a modified two-stream instability is discussed. It saturates first by the formation of electron phase space holes. The relativistic electron clouds modulate the ion beam and a secondary, spatially localized electrostatic instability grows, which saturates by forming a relativistic ion phase space hole. It accelerates electrons to ultra-relativistic speeds. (3) A simulation is also revised, in which two clouds of an electron-ion plasma collide at the speed $0.9 \mathrm{c}$. The inequal densities of both clouds and a magnetic field that is oblique to the collision velocity vector result in waves with a mixed electrostatic and electromagnetic polarity. The waves give rise to growing corkscrew distributions in the electrons and ions that establish an equipartition between the electron, the ion and the magnetic energy. The filament-, phase space hole- and corkscrew structures are discussed with respect to electron acceleration and magnetic field generation.
\end{abstract}

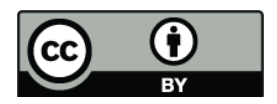

Correspondence to: M. E. Dieckmann (mark.e.dieckmann@itn.liu.se)

\section{Introduction}

Accreting compact objects such as neutron stars and black holes are a source of energetic plasma flows. The accreted plasma originates from a companion star in the case of microquasars (MQs) (Böttcher, 2007) and from the progenitor star of the compact object for gamma-ray bursts (GRBs) (Medvedev and Loeb, 1999; Piran, 2004; Fox and Meszaros, 2006). Gas and plasma clouds are engulfed by the huge black holes in the centres of galaxies (Melia and Falcke, 2001). Their mass accretion is driving active galactic nuclei (AGNs). As the plasma is attracted towards the compact object, it is accelerated to relativistic speeds. The infalling material typically forms an accretion disc around the compact object (Shakura and Sunyaev, 1973). Plasma thermalization processes within the accretion disc are thought to trigger instabilities (Balbus and Hawley, 1998), which accelerate charged particles and launch jets, e.g. through shocks (Das et al., 2003).

The ejection of jets by the MQs in our galactic neighbourhood, such as GRS 1915+105 or Cygnus X-3, can be observed directly (Fender and Belloni, 2004; Lindfors et al., 2007), which makes them suitable for systematic studies of the dynamical processes that occur in the jet and those that are responsible for its ejection in the first place. The coupled disc-jet system is not stationary in time and the accretion disc emissions and the jet speed and structure vary. The steady jet ejected by GRS 1915+105 moves at nonrelativistic or weakly relativistic speeds. Its transient jets reach Lorentz factors of a few, which are comparable to those of the collimated AGN jets (Harris and Krawczynski, 2002; Brunetti et al., 2002). Lorentz factors of several hundred are reached by the jets, which are ejected by imploding large stars during a long GRB (Piran, 2004; Waxman, 2006).

The jets are sources of electromagnetic radiation over a broad frequency band, some of which is attributed to synchrotron radiation (Lindfors et al., 2007; Harris and

Published by Copernicus Publications on behalf of the European Geosciences Union and the American Geophysical Union. 
Krawczynski, 2006; Piran, 2004; Gruzinov and Waxman, 1999). The AGN jets reveal the sources of this radiation, which are the knots and the hot spots (Brunetti et al., 2002). These sites convert flow energy into heat. This energy transfer could also be the origin of the synchrotron emissions, which we observe from the MQ jets (Lindfors et al., 2007) and during the prompt phase and the afterglow of long GRBs (Piran, 2004; Gruzinov and Waxman, 1999). The collisionless shocks accelerate the electrons and they amplify the magnetic field (Lindfors et al., 2007; Brunetti et al., 2002; Medvedev and Loeb, 1999; Pe'er and Zhang, 2006). The shocks occur, because plasma clouds collide within the jet (internal shocks) or because the jet impacts onto the stationary plasma of the interstellar medium (external shocks).

In the post-shock or downstream region of internal shocks in the jets of AGNs and GRBs, the magnetic energy density is thought to be close to that of the electrons (Harris and Krawczynski, 2002; Waxman, 2006). Both these energy densities should also be high to explain the synchrotron outbursts from the shocked MQ jet plasma (Lindfors et al., 2007). The relevance of the ion energy is not yet clear. The observations of the emission spectra of the jets of AGNs, for example, indicate that the ions are not directly involved (Harris and Krawczynski, 2002). However, if the shocks form by the collision of plasma clouds at mildly relativistic speeds, only the massive ions carry with them enough energy to accelerate electrons to highly relativistic speeds and to generate strong magnetic fields. We thus assume that the jets have a significant baryonic component and that a substantial amount of energy must be transferred from the ion flow to the electrons and to the magnetic field, in order to give rise to synchrotron eruptions. The release of ion kinetic energy on a kinetic (micro-)scale does probably not contradict the suggestion by Harris and Krawczynski (2002), that the ions do not establish an energy equilibrium with the electrons and the magnetic fields on the large scales they consider.

Identifying the relevant electron acceleration processes and magnetic field amplification mechanisms close to shocks and quantifying the peak magnetic field strengths and lifetimes is the key for a better understanding of the electromagnetic emissions of astrophysical jets. Apart from their importance for the radiation generation within jets, the shocks that move through the accretion disc may also contribute fast particles to the jet or to the hot disc halo (Das et al., 2003).

The global shock evolution takes place on magnetohydrodynamic scales, while the formation of the shock boundary and the energy dissipation mechanisms within this transition layer are attributed to kinetic processes. The coupling of the shock dynamics across both spatio-temporal scales complicates its full understanding (Siewert and Fahr, 2007). Even numerical simulations cannot tackle both scales simultaneously and they model idealized cases.

We review here some aspects of the formation of collisionless shocks, of the resulting particle acceleration and plasma thermalisation and of the magnetic field generation. Our fo- cus is on recent developments in the modelling of relativistic shocks and their foreshock with particle-in-cell (PIC) simulations. The small spatio-temporal scales that are accessible to PIC simulations and the constraints on the initial conditions and processes that can be simulated implies, that such simulations can often not be directly applied to explain experimental observations. PIC simulations can, however, give important qualitative insight into the dynamics of relativistic plasma and they can reveal novel instabilities and processes that may occur in energetic astrophysical plasmas.

The focus of this review paper is on the plasma phase space structures that give rise to magnetic field generation and particle acceleration. We refer to the Refs. (Lembege et al., 2004; Silva, 2006) for a review of global shock physics and simulations. By selecting appropriate initial and boundary conditions, the phase space structures can be isolated and examined in detail. Studies of these isolated structures are not directly related to the time-evolution of shocks. They can, however, give insight into the magnetic field strengths and the maximum particle energies that can be reached in a kinetic plasma under idealized conditions, because the considered structures play a key role in the dynamics of collisionless shocks. These estimates can, in turn, be compared to the observed magnetic field strengths and bulk plasma energies that are thought to be representative for the jets of accreting compact objects. These jet flows and their internal shocks can have characteristic bulk speeds that cover a wide range of speeds as we go from MQ jets through AGN jets to GRB jets. Parametric studies of the elementary phase space structures permit us to assess how the peak magnetic field strength and the possible particle acceleration scales, for example, with the initial beam speed or composition. This scaling can, potentially, be compared with observations of the synchrotron emissions of astrophysical jets and it can reveal interesting initial conditions for global shock simulations.

Section 2 explains the PIC simulation method and it revises some initial conditions that are often used for simulations in an astrophysical context. Section 3 discusses firstly the Weibel and filamentation instabilities, which are invoked as a magnetic field generation mechanism. Secondly, the electrostatic phase space holes are revised that result out of two-stream instabilities or the Buneman instability. The phase space holes are efficient in thermalising relativistic plasma flows. Section 4 demonstrates how plasma clouds, which collide in the presence of a magnetic field with a direction that is oblique to the collision direction, can establish an energy equilibrium between the ions, the electrons and the magnetic field. Section 5 summarizes the paper. 


\section{Particle-in-cell method and initial conditions}

\subsection{Particle-in-cell method}

A collisionless plasma can be described by the relativistic Vlasov equation and by the Maxwell's equations. The Vlasov equation evolves in time the probability distribution $f_{i}(\boldsymbol{x}, \boldsymbol{p}, t)$ of the phase space fluid, which is representing a plasma species $i$ under the influence of the electric and magnetic fields $\boldsymbol{E}$ and $\boldsymbol{B}$. These fields are evolved using the charge density $\rho_{t}(\boldsymbol{x})=\sum_{i} \rho_{i}(\boldsymbol{x})$ and the current $\boldsymbol{j}_{t}(\boldsymbol{x})=\sum_{i} \boldsymbol{j}_{i}(\boldsymbol{x})$. With $\Gamma(\boldsymbol{v})=\left(1-\boldsymbol{v}^{2} / c^{2}\right)^{-1}$ and $\boldsymbol{p}=m_{i} \Gamma(\boldsymbol{v}) \boldsymbol{v}$ the current and charge can be calculated as $\boldsymbol{j}_{i}(\boldsymbol{x})=q_{i} \int \boldsymbol{v} f_{i}(\boldsymbol{x}, \boldsymbol{v}, t) d^{3} \boldsymbol{v}$ and $\rho_{i}(\boldsymbol{x})=q_{i} \int f_{i}(\boldsymbol{x}, \boldsymbol{v}, t) d^{3} \boldsymbol{v}$. The equations are

$$
\begin{aligned}
& \frac{\partial f_{i}}{\partial t}+\boldsymbol{v} \cdot \nabla_{x} f_{i}+q_{i}(\boldsymbol{E}+\boldsymbol{v} \times \boldsymbol{B}) \cdot \nabla_{p} f_{i}=0, \\
& \nabla \times \boldsymbol{E}=-\frac{\partial \boldsymbol{B}}{\partial t}, \\
& \nabla \times \boldsymbol{B}=\mu_{0} \epsilon_{0} \frac{\partial \boldsymbol{E}}{\partial t}+\mu_{0} \boldsymbol{j}_{t}, \\
& \nabla \cdot \boldsymbol{E}=\rho_{t} / \epsilon_{0}, \nabla \cdot \boldsymbol{B}=0,
\end{aligned}
$$

where $m_{i}$ and $q_{i}$ are the nonrelativistic mass and the charge of the species $i$. The $\epsilon_{0}, \mu_{0}$ and $c$ are the permittivity, the permeability and the vacuum speed of light, respectively.

Applying the method of characteristics replaces the Vlasov-Maxwell equations by those of Klimontovich and Dupree (Dupree, 1963). This approach leads to the particle-in-cell method, which resolves all kinetic waves. PIC simulations define $\boldsymbol{E}(\boldsymbol{x})$ and $\boldsymbol{B}(\boldsymbol{x})$ and the macroscopic current $\boldsymbol{j}_{i}(\boldsymbol{x})$ on a grid and they substitute $f_{i}(\boldsymbol{x}, \boldsymbol{p}, t)=\sum_{k=1}^{N} S\left(\boldsymbol{x}-\boldsymbol{x}_{k}[t]\right) \delta\left(\boldsymbol{p}-\boldsymbol{p}_{k}[t]\right)$. Each computational particle (CP) with the index $k$ of the species $i$ is specified by a position $\boldsymbol{x}_{k}(t)$ and a momentum $\boldsymbol{p}_{k}(t)$, by its charge and mass $q_{c p}$ and $m_{c p}$ and by a spatial shape function $S(\boldsymbol{x})$. The $q_{c p}$ and $m_{c p}$ of each CP can differ from those of the physical particles it represents. The charge-to-mass ratio has to be preserved. A PIC code solves the equations

$$
\frac{d \boldsymbol{p}_{c p}}{d t}=q_{c p}\left(\boldsymbol{E}\left[x_{c p}\right]+\boldsymbol{v}_{c p} \times \boldsymbol{B}\left[x_{c p}\right]\right), \frac{d \boldsymbol{x}_{c p}}{d t}=\boldsymbol{v}_{c p}
$$

to update the position $x_{c p}$ and momentum $p_{c p}$ of each CP. The $\boldsymbol{E}, \boldsymbol{B}$, which are defined on the grid, have to be interpolated to $\boldsymbol{x}_{c p}$. The microcurrents $q_{c p} v_{c p}$ of all CPs have to be interpolated to the grid to give the macroscopic $j_{i}$. The interpolation scheme is determined by the shape function. In many PIC simulation schemes the Eqs. (2) and (3) are used to evolve the electromagnetic fields, while the Eq. (4) is either fulfilled as a constraint, as it is the case for our algorithm (Eastwood, 1991), or it is enforced by correction steps. The particle-in-cell method is detailed in the review paper by Dawson (1983) or in the books by Hockney and Eastwood (1989) and Birdsall and Langdon (1991).

\subsection{Initial conditions}

PIC codes have been used now for decades to tackle problems in collisionless plasma physics. Early groundbreaking simulation work has been accomplished in the seventies of the last century. Electrostatic phase space holes, which constitute the nonlinearly saturated final state of the two-stream instability (Roberts and Berk, 1967), have been found and explored in higher dimensions (Morse and Nielson, 1969) and in relativistic regimes (Thode and Sudan, 1973). The filamentation instability has been examined by Morse and Nielson (1971) with 1D and 2-D PIC simulations. The formation and the structure of electrostatic shocks has also been addressed (Forslund and Shonk, 1970; Forslund and Freidberg, 1971).

PIC simulations have modelled colliding plasmas in one dimension (1-D) (Chapman et al., 2005; Matsukiyo and Scholer, 2006; Schmitz et al., 2002; Hoshino and Shimada, 2002; Lembege and Dawson, 1987, 1989; Sorasio et al., 2006; Bessho and Ohsawa, 1999, 2000) or in 2-D or 3D (Lembege et al., 2004; Dieckmann et al., 2006b, 2008; Frederiksen et al., 2004; Hededal et al., 2004; Hededal and Nishikawa, 2005; Jaroschek et al., 2004; Hellinger et al., 2007; Spitkovsky, 2008a; Chang et al., 2008; Kato, 2007; Kazimura et al., 1998; Langdon et al., 1988). Interpenetrating plasmas are considered by Shimada and Hoshino (2002); Silva et al. (2003); Fonseca et al. (2003); Lapenta et al. (2007). The shock physics is three-dimensional. PIC simulations have not yet been performed in 3-D and for high ion-toelectron mass ratios. The 1-D and 2-D PIC simulations are nevertheless useful. The 1-D simulations can resolve the interplay of electrons and ions at the correct mass ratio, with a good statistical plasma representation and their low computational cost permits parametric studies. The more realistic 2D PIC simulations have to employ reduced mass ratios, even though the largest ones are approaching now the full mass ratio (Spitkovsky, 2008a). The 2-D PIC simulations can provide important insights into the shock structuring orthogonal to the flow direction, but they can probably not resolve the non-linear evolution of the shock front correctly, i.e. the filament merging, which requires two dimensions orthogonal to the flow velocity vector (Morse and Nielson, 1971).

Interpenetrating plasmas typically involve spatially uniform beam distributions and periodic simulation boundary conditions. Such simulations can show how plasma instabilities saturate and what secondary processes they can trigger. Figure 1a illustrates the model that is often invoked to motivate such PIC simulations in the context of astrophysical plasma shocks. Shock-reflected ion beams are frequently observed at the Earth's foreshock (Eastwood et al., 2005) and are thought to be present also in its astrophysical counterparts. Indeed, PIC simulations find shock-reflected ion beams at very high Mach-number shocks (Sorasio et al., 2006) and at mildly relativistic shocks (Bessho and Ohsawa, 1999, 2000; Dieckmann et al., 2008). 


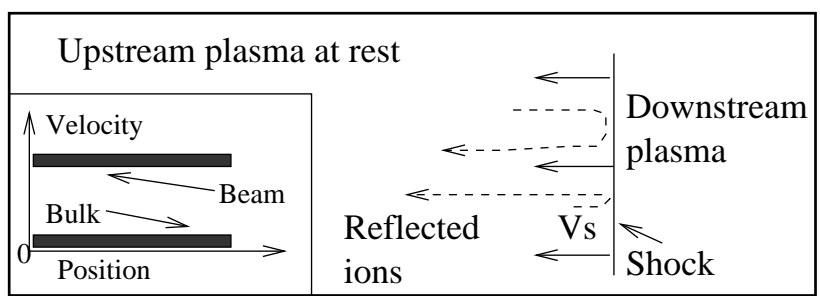

Panel a: Interpenetrating beams

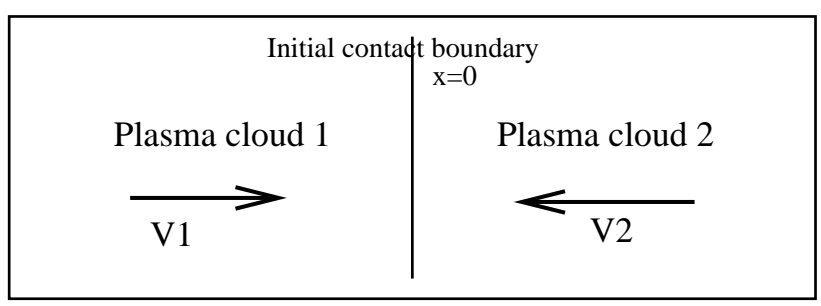

Panel b: Colliding plasmas

Fig. 1. (a) A shock moves with the speed $v_{s}$ to the left through a plasma at rest. It reflects a fraction of upstream particles (here ions denoted by dashed arrows), which move at the speed $v>v_{s}$ and outrun the shock. The simulation box is placed in the upstream region away from the shock. The inset shows the phase space distribution, which is the initial simulation plasma. The upstream (bulk) plasma has $v \approx 0$ and the reflected ions form the beam. Both beams are taken for simplicity to be spatially uniform and periodic in space and their thermal spread is low. (b) Two plasma clouds collide at the initial contact boundary $x=0$ with the oppositely directed flow velocity vectors $\boldsymbol{v}_{1}$ and $\boldsymbol{v}_{2}$. The piston method introduces a reflecting boundary at $x=0$.

Many simulations consider the collision of two plasma clouds at the initial contact boundary $x=0$, which is displayed by the Fig. 1b. The clouds can have different densities (Bessho and Ohsawa, 1999, 2000; Sorasio et al., 2006; Dieckmann et al., 2008) or magnetisations. Often the clouds are equally dense and they differ only in the direction, but not in the magnitude, of their flow velocity vector. This gives $\boldsymbol{v}_{1}=-\boldsymbol{v}_{2}$ in Fig. 1b. Then the system is symmetric relative to $x=0$. The piston method exploits this symmetry by introducing a reflecting boundary at $x=0$, which cuts in half the necessary computations. The inflowing plasma is reflected at $x=0$ and the counterstream results in a shock that moves away from the boundary. Two similar shocks would form without the reflecting boundary. The piston method works well for nonrelativistic $\left|\boldsymbol{v}_{1}\right|$ and electrostatic (Forslund and Shonk, 1970; Forslund and Freidberg, 1971) plasmas, or if the magnetic field is orthogonal to $\boldsymbol{v}_{1}$ (Langdon et al., 1988; Chapman et al., 2005; Matsukiyo and Scholer, 2006). It must be verified though that the relevant instabilities in the system have wavevectors parallel to the flow velocity vector, otherwise the reflection at the boundary may change the spectrum of unstable waves.

\section{Particle acceleration or magnetic field generation}

In this section we summarize some studies that are relevant for a better understanding of particle acceleration or magnetic field generation in energetic astrophysical plasmas. While the discussed instabilities and nonlinear structures can provide both, the limited computer time does typically not (yet) allow to examine both aspects simultaneously.

\subsection{Magnetic field generation}

Kinetic instabilities in a spatially uniform plasma that yield growing magnetic fields are the Weibel instability (Weibel, 1959) due to a thermal anisotropy and the filamentation instability (Molvig, 1975), which is driven by particle beams. Selecting beams with a thermal anisotropy gives a cumulative instability (Lazar et al., 2006).

The most unstable wavenumbers $\boldsymbol{k}$ of the Weibel instability are aligned with the direction, in which the particle temperature is at its lowest (Tzoufras et al., 2007). This instability has been addressed by many authors (Yoon and Davidson, 1987; Davidson et al., 1972; Yoon, 2007; Shukla, 2007). The filamentation instability is beam-driven and it has been considered with or without a guiding magnetic field. It is attracting a renewed interest due to its importance in astrophysics and inertial confinement fusion. Its most unstable $\boldsymbol{k}$ is oriented orthogonally to the beam velocity vector.

The filamentation instability competes with the twostream modes and the mixed modes (Bludman et al., 1960; Bret et al., 2004; Dieckmann et al., 2006; Tzoufras et al., 2006). The filamentation instability is dominant if the beam speeds are relativistic, if the two beams have a similar density and if they consist of the same particle species (Bret, Gremillet and Bellido, 2007). A magnetic field that is aligned with the beam flow direction can reduce its growth rate and even suppress it (Bret et al., 2006; Stockem et al., 2008). The magnetic field strength that suppresses the partially electrostatic mixed modes is higher than that for the electromagnetic filamentation instability, if the beams move at relativistic speeds (Bret et al., 2006). The electrostatic two-stream instability is unaffected by a flow-aligned $\boldsymbol{B}$ field. Increasing $|\boldsymbol{B}|$ thus shifts the unstable wave spectrum from electromagnetic to electrostatic.

The Weibel instability driven by a thermal anisotropy in a spatially uniform electron distribution has been examined with a 3-D PIC simulation by Romanov et al. (2004) who found that the growing magnetic fields feed on the thermal anisotropy and form complicated helical structures. Few PIC simulation studies of thermal-anisotropy driven instabilities have been performed in comparison with simulation studies of the filamentation instability, which has been examined in 1-D (Morse and Nielson, 1971; Califano et al., 2002; Rowlands et al., 2007), in 2-D (Morse and Nielson, 1971; Honda et al., 2000; Dieckmann et al., 2007; Medvedev et al., 2005; Stockem et al., 2008) and in 3D (Fonseca et al., 2003; Silva 
et al., 2003; Califano et al., 2006; Jaroschek et al., 2004). These simulations addressed the filamentation instability for a wide range of initial conditions such as the speed or the constituents (electrons, positrons and ions).

The filamentation instability and the Weibel instability evolve similarly. The magnetic field component of the growing waves spatially separates the moving charged particles, which carry currents of opposite polarity. This separation channels the particles into flux tubes. The characteristic size of the flux tubes is the electron skin depth $\lambda_{e}=c / \omega_{p}$, where $\omega_{p}=\left(n_{e} e^{2} / m_{e} \epsilon_{0}\right)^{(1 / 2)}$ is the plasma frequency of electrons with the number density $n_{e}$. The net current of these forming flux tubes further amplifies the magnetic field.

One form of the nonlinear evolution of the filamentation instability is well-understood for spatially uniform beams of counter-propagating electrons (Rowlands et al., 2007; Stockem et al., 2008). These simulations are of the type displayed in Fig. 1a. As the current channels develop for this form of the filamentation instability, the electron density is spatially modulated. Electrostatic fields try to restore the local charge neutrality of the plasma. Eventually, this restoring force cancels any further wave growth. This limits the peak magnetic energy to about $10 \%$ of the beam kinetic energy, which has also been reported for the final state of counterpropagating electron-positron beams (Silva et al., 2003). The current channels, which constitute the final state of the filamentation instability, interact through electromagnetic forces and the channels merge (Bogdan and Lerche, 1985; Medvedev et al., 2005).

Figure $2 \mathrm{a}$ and $\mathrm{b}$ show the electron current, when the filamentation instability has just saturated and after the flux tubes have undergone many mergers. It corresponds to Fig. 6 in (Dieckmann et al., 2007), where simulation details are discussed. The simulation resolves the $\mathrm{x}-\mathrm{y}$ plane and uses periodic boundary conditions. The two electron beams stream at equal speed moduli in opposite z-directions. Initially, the electron beams were spatially uniform.

These mergers dissipate the free energy and thermalise the plasma, which limits the life-time of the magnetic field structures. The electrons are not strongly accelerated. The electric fields due to the electron displacement are, however, strong enough to displace ions and to heat them (Honda et al., 2000; Califano et al., 2002).

Another saturation mechanism has been proposed by Davidson et al. (1972). The growing magnetic fields modify the electron trajectories. Eventually, the electrons are magnetically trapped. The saturation of the Weibel instability through magnetic trapping sets in, once the electron gyrofrequency due to the generated magnetic fields becomes comparable to the value of the linear growth rate. Califano et al. (2002) have also observed this process.

PIC simulation studies also exist that address the collisions of plasma clouds at relativistic speeds, which correspond to the case sketched out in Fig. 1b. The plasma parameters like
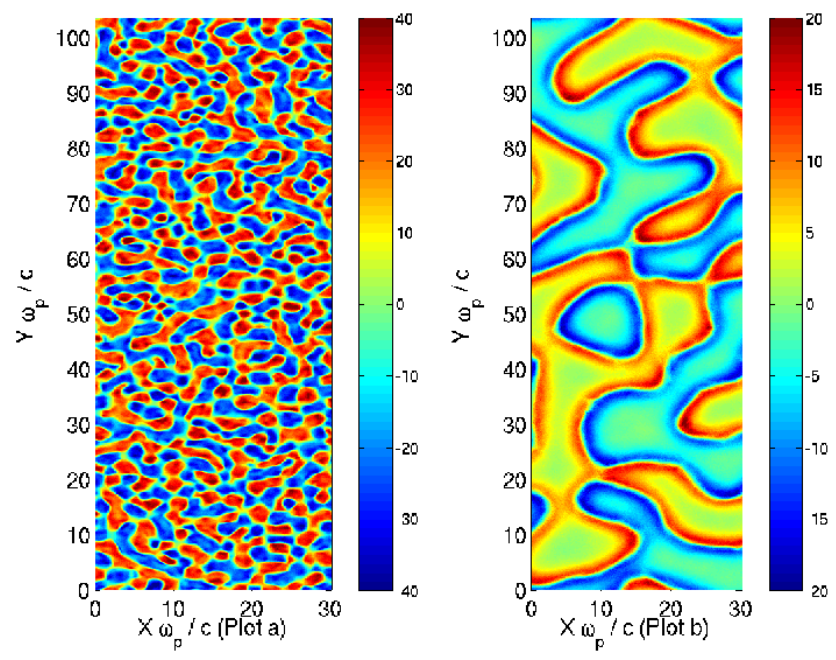

Fig. 2. (a) shows the current distribution along the beam flow (zdirection in a part of the simulation box, when the filamentation instability has just saturated. (b) shows the same current distribution at a later time, when the current filaments have undergone many mergers. The electron skin depth is the unit for $\mathrm{x}$ and $\mathrm{y}$.

the strength of a flow-aligned magnetic field or the density ratio of the colliding plasma clouds determine, which wave mode is dominant. The solution of the linear dispersion relation for relativistic beams of electrons in unbounded plasma can guide us at least qualitatively in this respect.

The interaction of a relativistic electron beam and the bulk electrons in unmagnetized plasma drives the two-stream instability, the mixed mode instability and the filamentation instability, if we neglect the ion motion (Bret et al., 2004). The two-stream instability grows too slowly for relativistic collisions and it is typically neglected. If both colliding plasma clouds have similar densities, as in the simulation by Nishikawa et al. (2006), it should be the filamentation instability that dominates the plasma dynamics (Bret, Gremillet and Bellido, 2007). The simulations by Nishikawa et al. (2006) show that the heated electron distributions decrease faster than exponential for large relativistic momenta, which is characteristic for the filamentation instability (Dieckmann et al., 2007). If the beam density ratio is 3 , which gives a larger relative growth rate for the mixed mode, the heated electron distribution can be approximated by a power-law $E^{-2.7}$, where $E$ is the relativistic electron energy (Hededal et al., 2004). The power-law decrease is similar to that observed for relativistic electrostatic two-stream instabilities (Dieckmann et al., 2006; Lapenta et al., 2007). This power-law decrease corresponds to a turbulent, marginally stable state (Treumann and Jaroschek, 2008). The electromagnetic filamentation mode may not excite such turbulent states, while the electrostatic component of the mixed mode does. 
A guiding magnetic field suppresses the filamentation instability and the mixed mode instability, if it is strong (Bret et al., 2006). This has been demonstrated by Hededal and Nishikawa (2005) for relativistic collision speeds and by Dieckmann et al. (2006b) for mildly relativistic collisions. The dominant mode is then initially the two-stream mode. Since the two-stream modes accelerate electrons only along the magnetic field, secondary anisotropy-driven Weibel instabilites (Yoon, 2007) can develop that overcome the magnetic confinement.

\subsection{Particle acceleration}

The magnetic field can confine the wavevectors and, thus, the plasma dynamics to one spatial dimension, which can be modelled with 1-D PIC simulations. This possibility had been proposed earlier (Thode and Sudan, 1973), which allowed these authors to examine a two-stream instability driven by relativistic electron beams. It is clear that this confinement is not perfect, because the growth rate of the mixed modes decreases slowly with an increasing $B_{0}$. The magnetic field may also not confine secondary modes that develop after the two-stream instability has saturated. The 1-D simulations of relativistic beam instabilities are thus not necessarily realistic, but they can give an important qualitative insight into the processes in relativistic plasma flows. We review here some works related to a relativistic electrostatic ion beam driven instability and we illustrate their findings.

The Buneman instability (Buneman, 1959) has been widely examined. A classification of two-stream and Buneman instabilities is given by Lapuerta and Ahedo (2002). It develops when unmagnetized electrons stream relative to the ions. A sequence of PIC simulations has analysed its nonlinear saturation and evolution as well as the ion evolution (Ishihara et al., 1980, 1981; Hirose et al., 1982). This instability has been considered further by many authors. Strictly speaking, the (nonrelativistic) Buneman instability develops between an electron beam and an ion beam with the same charge density modulus. The instability we consider here differs from the Buneman instability but its evolution is similar. We refer to this instability as a Buneman-type instability (BTI).

We consider a proton beam, which moves with the speed $v_{b}$ to increasing values of $x$, with the number density $n_{b}$ and the plasma frequency $\omega_{b}=\left(n_{b} e^{2} / m_{p} \epsilon_{0}\right)^{1 / 2}$. The electrons are at rest and have the number density $n_{e}$ and $\omega_{p}=\left(n_{e} e^{2} / m_{e} \epsilon_{0}\right)^{1 / 2}$, which we set to $\omega_{p}=10^{5}(2 \pi / s)$. The $\boldsymbol{B}=0$. The charge density of the electron beam and the proton beam are equal in their respective rest frame. In the rest frame of the electrons $\omega_{p}^{2}=m_{p} \omega_{b}^{2} / m_{e} \Gamma\left(v_{b}\right)$ with $m_{p} / m_{e}=1836$. This ratio of $n_{b} / n_{e}$ equals that in the simulation by Dieckmann et al. (2004). The electron thermal speed is defined as $v_{t e}=\left(k_{B} T_{e} / m_{e}\right)^{1 / 2}$ and set to $v_{t e}=10^{7} \mathrm{~m} / \mathrm{s}$. The beam thermal speed $v_{t b}=\left(m_{e} / m_{p}\right)^{1 / 2} v_{t e}$. The Boltzmann constant is $k_{B}$ and $T_{e}$ is the electron temperature. The pro- ton beam moves with the speed $v_{b}=0.99 \mathrm{c}$. A second proton beam with the same density as the first beam moves with $-v_{b}$ along the $\mathrm{x}$-direction to enforce current neutrality. Its thermal speed is $v_{t b} / 10$. This lower temperature and, thus, the lower initial thermal noise levels delay the onset of the BTI, while both beam temperatures are too low to yield significant differences in the linear growth rates of the respective BTIs. An asymmetry is established between the BTIs driven by both beams.

An almost identical setup is used by Dieckmann et al. (2004). Here we introduce an additional heavy ion component with $m_{d}=8 m_{p}$ and the charge $-e$. This species compensates the excess charge of the proton beams and replaces the immobile negative charge in the previous work. This mobile component leaves the simulation results unchanged and participates in the plasma dynamics only at the simulation's end. The system of four beams is not necessarily realistic, but it reveals an aspect of the BTI that may occur in energetic astrophysical plasmas.

The simulation box with periodic boundary conditions has the length $L=N_{g} \Delta_{x}$, where $N_{g}=3 \times 10^{4}$ and the grid cell size is $\Delta_{x}=10 \mathrm{~m}$ or $\Delta_{x}=0.63 \lambda_{D}$, where the Debye length $\lambda_{D}=v_{t e} / \omega_{p}$. Each plasma species is represented by $N_{p} \approx 10^{7} \mathrm{CPs}$, respectively. The dispersion relation of the BTI driven by one proton beam and by one electron beam is in the reference frame of the electrons

$\frac{\omega_{p}^{2}}{\omega^{2}}+\frac{\omega_{b}^{2}}{\Gamma^{3}\left(v_{b}\right)\left(\omega-k v_{b}\right)^{2}}=1$.

The scalar $\omega$ and $k$ are the wave frequency and the wavenumber in the $\boldsymbol{x}$-direction, respectively. The fastestgrowing wave has the frequency $\omega_{u} \approx \omega_{p}$ and the wavenumber $k_{u} \approx \omega_{p} / v_{b}$. Its phase speed is thus $v_{p h}=\omega_{u} / k_{u} \approx v_{b}$ or $v_{p h} \approx c$ for relativistic $v_{b}$. Its wavelength $\lambda_{u} \approx 2 \pi \lambda_{e}$ with $\lambda_{e}=c / \omega_{p}$. The wave spectrum is well-resolved by the simulation box length of $L=100 \lambda_{u}$. The exponential growth rate of this mode is $\omega_{i} / \omega_{p} \approx \sqrt{3}\left(m_{e} n_{b} / m_{p} n_{e}\right)^{1 / 3} / 2^{4 / 3} \Gamma\left(v_{b}\right)$.

The BTI saturates by the electrostatic trapping of electrons and ions (Roberts and Berk, 1967; Lancellotti and Dorning, 2003; Luque and Schamel, 2005; Eliasson and Shukla, 2005, 2006). The electric fields and the particles reach a dynamical equilibrium, in which the wave growth is halted and a meta-stable state is established. This can be shown using the relevant energy densities. The box-averaged electrostatic energy density $E_{E}(t)=N_{g}^{-1} \sum_{j=1}^{N_{g}} \epsilon_{0} E_{x}^{2}(j \Delta x, t)$ and the box-averaged electron kinetic energy density $E_{K}(t)=\left(m_{c e} c^{2} / N_{g} \Delta_{x}^{3}\right) \sum_{j=1}^{N_{p}}\left(\Gamma\left[\boldsymbol{v}_{j}\right]-1\right)$. Both energy densities are shown in Fig. 3. The summation is over the $N_{g}$ grid cells and over the $N_{p}$ CPs with the mass $m_{c e}$ that represent the electrons. The energy densities are normalized to the initial electron thermal energy density $E_{0}=E_{K}(t=0)$.

The plasma phase space distribution forms a chain of trapped particle islands with the period $\lambda_{u}$. If the electrostatic potential is stationary in the reference frame that moves with 


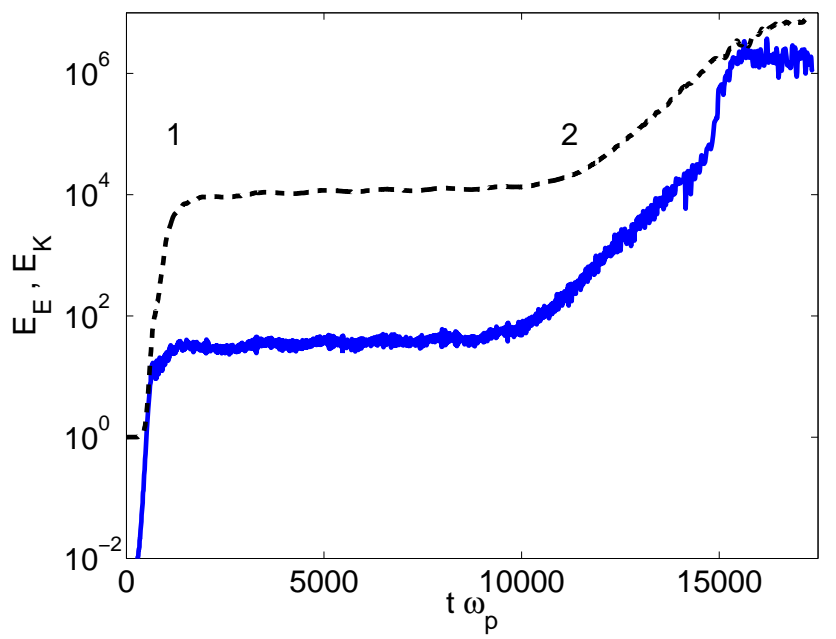

Fig. 3. Electric $E_{E}$ (blue lower curve) and electron kinetic energy densities $E_{K}$ (dashed upper curve) in units of $E_{0}$ : Initially, only $E_{E}(t)$ grows exponentially. At $t \omega_{p} \approx 500$ the electrons are accelerated, as $E_{K}$ evidences. Both curves saturate at $t \omega_{p} \approx 10^{3}(1)$ and a meta-equilibrium is established. After $t \omega_{p} \approx 11000$ (2) both curves grow again and saturate at the simulation's end.

$v_{p h}$, then the potential energy plus the kinetic energy of the particle is conserved along a trajectory inside the trapped particle island. The potential often reaches a steady state after some initial oscillations around an equilibrium amplitude.

Figure 4 shows the electron and proton phase space distribution at the time $t \omega_{p}=1440$.

The proton beam oscillates with the period $\lambda_{u}$. The oscillation in the interval $0<x / \lambda_{u}<1$ is approximately sinusoidal, whereas the second one has a steeper slope around $x / \lambda_{u} \approx 1.3$. The electron distribution consists of a bulk population with $p_{x} \approx 0$ and of fast beams. The electron beam in the interval $0<x / \lambda_{u}<0.5$ is oscillatory, but the oscillation period is $\lambda_{u} / 2$. The separation between neighboring trapped particle islands is still $\lambda_{u}$, but they are asymmetric with respect to the centre of the trapped particle island, which should be at $x \approx \lambda_{u} / 2$ in Fig. $4 \mathrm{~b}$. This contrasts the case of the BTI driven by a tenuous beam (Dieckmann et al., 2007) that saturates by forming symmetric electron phase space holes. The lower growth rate $\omega_{i}$ of the BTI considered by Dieckmann et al. (2007) presumably allows for a smoother nonlinear saturation, which gives symmetric phase space holes. Indeed, relativistic electron phase space holes are sensitive against perturbations and easily deform (Eliasson and Shukla, 2005). The electron beam in the interval $x / \lambda_{u}>1$ shows an oscillation with the period $\lambda_{u}$, but the electrons do not follow a closed path. Figure $4 \mathrm{~b}$ also shows electron beams with $p_{x}<0$. These electrons have been trapped by a wave moving with $v_{p h}=-\omega_{u} / k_{u}$. This wave is driven by the second proton beam and by back-scattering of wave energy from the stronger wave with $v_{p h}=\omega_{u} / k_{u}$.
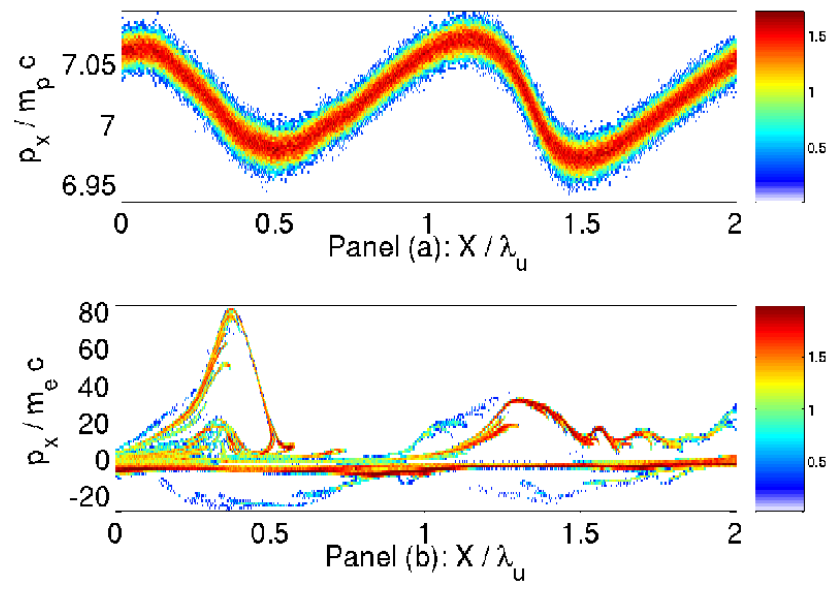

Fig. 4. (a) The phase space distribution of the beam protons in a sub-interval of the simulation box at the time $t \omega_{p}=1440$. (b) The electron phase space distribution sampled in the same sub-interval at the same time. The period of the islands of trapped electrons is $\lambda_{u} \approx 2 \pi \lambda_{e}$, in line with that of the wave. The 10-logarithmic colorscale is in units of a CP.

The peak electron energy in Fig. $4 \mathrm{~b}$ is in line with what we expect. The trapped electrons oscillate around the extremum of the wave potential that is a stable equilibrium point for the electron phase space trajectory. This equilibrium point moves with $v_{p h} \approx v_{b}$. Since the electrons are picked up by the potential from the thermal bulk population at a speed $v_{\min } \ll c$, their peak speed in the electron rest frame reaches $v_{\max } \approx 2 v_{b} /\left(1+v_{b}^{2}\right)$. This yields a Lorentz factor $\Gamma\left(v_{\max }\right) \approx 2 \Gamma^{2}\left(v_{b}\right)-1$. We expect a maximum $\Gamma\left(v_{\max }\right) \approx 100$ with $\Gamma\left(v_{b}\right)=7.09$.

Figure 3 displays that this equilibrium between the wave energy and the electron kinetic energy is stable over $10^{4} / \omega_{p}$, after which a new exponential growth phase of $E_{E}(t)$ sets in. We consider the relevant phase space distribution at the time $t \omega_{p}=10^{4}$.

Figure 5 shows that two periods of the beam oscillations have merged to a single large one. This structure is spatially correlated with an electron depletion. A dense cloud of mildly relativistic electrons moves ahead of the perturbation. It is thought that this cloud accelerates the protons through a mechanism similar to wakefield acceleration (Dieckmann et al., 2006a). This structure is self-amplifying and its consequence is the exponential increase of $E_{E}(t)$ in Fig. 3 at late times.

Relativistic structures in the proton distribution develop, which accelerate the electrons to ultrarelativistic speeds. Figure 6 displays the phase space distributions of the electrons and of both proton beams at the simulation's end at $t \omega_{p}=17300$. 

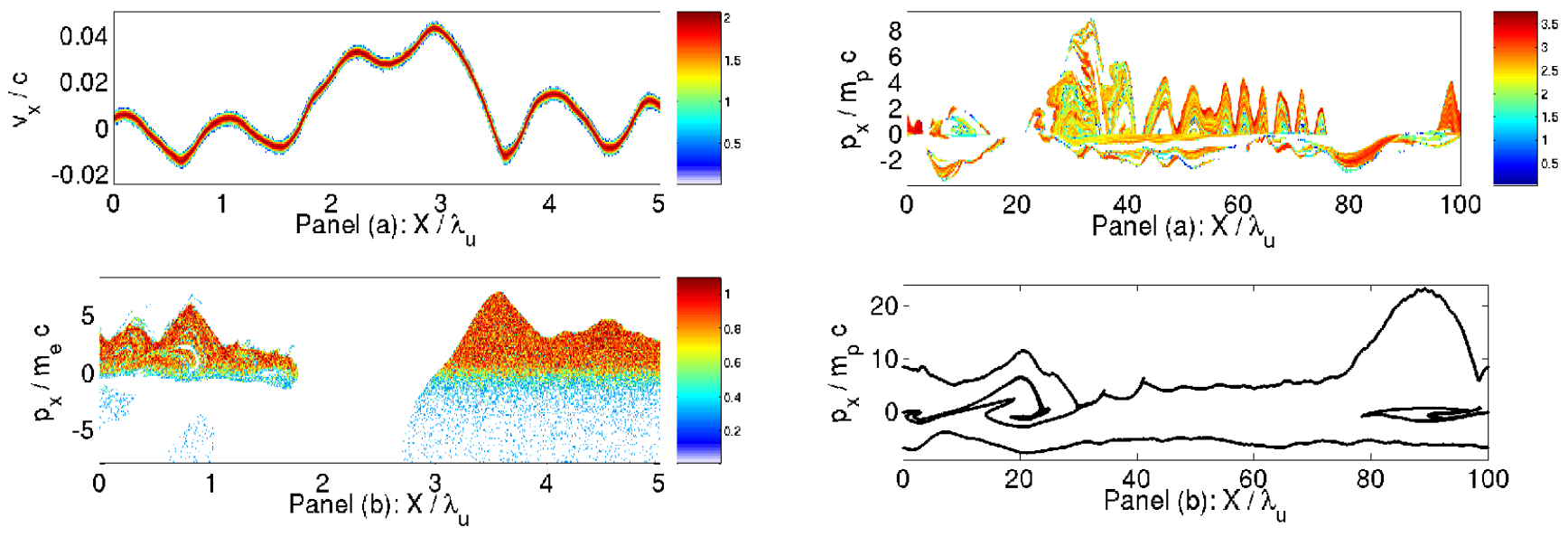

Fig. 5. (a) The phase space distribution of the beam protons in a sub-interval of the simulation box at $t \omega_{p}=10^{4}$. The beam shows a large oscillation for $1.5<x / \lambda_{u}<3.5$. (b) The electron phase space distribution in the same interval. The large proton structure coincides with an electron void. Both distributions are measured in the beam frame moving with the speed $v_{b}=0.99 c$ and the colour denotes the 10-logarithm of the number of CPs.

The phase space reveals clouds of electrons, that reach peak Lorentz factors of $\Gamma=8 m_{p} / m_{e}$. The highest electron energies are reached just ahead of the relativistic proton phase space vortex at $x / \lambda_{u} \approx 20$. The extreme electrostatic field of this phase space hole is a strong electron accelerator. At this time the proton vortex is still driven by the conversion of the directed proton beam energy to the accelerating electrons and protons. The second vortex at $x / \lambda_{u} \approx 90$ is connected to the main vortex across the periodic boundary. The introduction of a magnetic field with $e|B| / m_{e} \omega_{p} \approx 0.015$ oriented at $45^{\circ}$ relative to $x$ has left the results unchanged (Dieckmann et al., 2006a). The interaction between the ultrarelativistic electrons and the electrostatic fields should result in significant electromagnetic radiation (Fleishman and Toptygin, 2007).

\section{Particle acceleration and magnetic field generation}

In this section we revise simulations of colliding plasma clouds carrying an oblique magnetic field. Early studies of nonrelativistic collisions have been performed by Lembege and Dawson (1989). The selection of unequal densities of the colliding plasma clouds and mildly relativistic speeds have then resulted in ultrarelativistic electrons in the simulations by Bessho and Ohsawa $(1999,2000)$ and also in magnetic fields with near-equipartition energies in the simulation by Dieckmann et al. (2008). The dominant structure discussed here thus lead simultaneously to a direct strong electron acceleration and magnetic field amplification, which contrasts

Fig. 6. (a) The electron phase space distribution at $t \omega_{p}=17300$. The electrons reach $\Gamma>m_{p} / m_{e}$. The colour is the 10-logarithm of the number of CPs. (b) The proton beams. The beam with $p_{x} / m_{p} c \approx-7$ is the cold proton beam inserted to cancel the net current. It is modulated but it does not form vortices. The proton beam that had initially $p_{x} / m_{p} c \approx 7$ forms vortices at $x / \lambda_{u} \approx 20$ and at $x / \lambda_{u} \approx 90$.

the cases in the previous section, which achieved only one of these two aspects, respectively.

The case study by Dieckmann et al. (2008) illustrates this setting. It has considered a relative flow velocity vector $\boldsymbol{v}_{b}=v_{b} \boldsymbol{x}$ between the clouds that is oblique to a background magnetic field $\boldsymbol{B}_{0}=B_{x} \boldsymbol{x}+B_{z} z$ with $B_{x} \gg B_{z}$. The similar studies by Bessho and Ohsawa $(1999,2000)$ have used a larger angle between the $\boldsymbol{v}_{b}$ and the magnetic field.

The flow-aligned magnetic field component in (Dieckmann et al., 2008) has suppressed the filamentation instability and the mixed mode instability, which has been tested with 2D PIC simulations. The $B_{z} \neq 0$ has introduced a new instability. The PIC simulation demonstrates that a wave is growing with a mixed electrostatic and electromagnetic polarity, possibly an oblique whistler (Tokar and Gary, 1985). We refer to this wave as a whistler-type wave. Its electrostatic component can couple it directly to the plasma flow. A good conversion from the kinetic to the wave energy is thus achieved. Since the electrostatic component is tied through the dispersion relation to an electromagnetic component, the flow energy is also coupled directly to the growing magnetic fields. In fact, an energy equi-partition between the ion kinetic energy, the electron kinetic energy and the magnetic field energy could be established behind the structure. Similar electron and ion energies have also been observed by Bessho and Ohsawa (1999, 2000).

Whistlers are efficient particle accelerators in Solar system plasmas and in astrophysical plasmas (Levinson, 1992; Kuramitsu and Krasnoselskikh, 2005; Roth, 2007). Whistlers with a strong wave magnetic field have been observed in 
laboratory settings (Stenzel et al., 2006). It is thus natural to think that whistlers can also be an important source of magnetic fields in astrophysics. Apart from being an efficient mechanism for converting kinetic to magnetic energy, the whistler-type instability can develop in plasmas with an electron thermal speed that is comparable to the speed difference of the colliding plasmas, which might be relevant for the plasmas in the GRB jets (Dieckmann et al., 2008; Pe'er et al., 2007).

In what follows we review the initial conditions similar to those used by Dieckmann et al. (2008), which correspond to the case depicted by Fig. 1b, and the results of this PIC simulation. Two plasma clouds collide at the position $x=0$, each consisting of the electrons and ions with the masses $m_{e}$ and $m_{i}=400 m_{e}$. Each cloud initially occupies half the simulation box. No further particles are inserted through the periodic boundaries during the simulation. The plasma frequency of the species $j$ with the number density $n_{j}$ is $\omega_{p, j}=\left(n_{j} q_{j}^{2} / m_{j} \epsilon_{0}\right)^{1 / 2}$. The electrons of the cloud 1 have $\omega_{p, 1}=\sqrt{2} 10^{5} \times 2 \pi / s$ and the ions $\omega_{p, 2}=\omega_{p, 1} / \sqrt{R}$. The cloud 1 (the dense cloud) is initially moving with the positive speed $v_{c}$ along the $x$-direction. The electrons and ions of the cloud 2 (the tenuous cloud) have $\omega_{p, 3}=\omega_{p, 1} / \sqrt{10}$ and $\omega_{p, 4}=\omega_{p, 3} / \sqrt{R}$, respectively. The tenuous cloud moves with the speed $-v_{c}$ along the $x$-direction. Initially, all mean speeds along the $y, z$ directions vanish. All plasma species have the temperature $T=100 \mathrm{keV}$. The positions $x, y$ have the units of the boxaveraged electron skin depth $\lambda_{s}=\sqrt{2} c /\left(\omega_{p, 1}^{2}+\omega_{p, 3}^{2}\right)^{1 / 2}$. The time has the unit $\lambda_{s} / c$. The clouds collide along the $\boldsymbol{x}$ at the speed $v_{b}=2 v_{c} /\left(1+v_{c}^{2} / c^{2}\right)=0.9 c$, representative of internal GRB shocks (Piran, 2004). The collision of two unmagnetized plasma clouds with a similar speed and density ratio modelled by Frederiksen et al. (2004) has evidenced the development of the filamentation instability on timescales $10<t<10^{2}$.

We introduce a spatially uniform oblique magnetic field. The magnetic field component with $e B_{x} / m_{e}=\omega_{p, 1}$ or $B_{x}=5 \mu \mathrm{T}$ along the $x$-axis suppresses the filamentation. We set $B_{y}=0$. The $B_{z}=B_{x} / 10$ enhances the energy dissipation. The plasma $\beta=P_{t h} / P_{b}$ quantifies the importance of the magnetic field. The thermal pressure $P_{t h}=n k_{B} T$ is a measure for the thermal energy in a plasma with the number density $n$ and the temperature $T$. The magnetic pressure is $P_{b}=B_{0}^{2} / 2 \mu_{0}$. The dense cloud has a $\beta \approx 1$. The $\beta$ is measured in the rest frame of a cloud and it determines the dispersive properties of the plasma but not necessarily its kinematic ones, which depend also on the flow energy. The initial box-averaged plasma kinetic energy density exceeds the magnetic field energy density by a factor $\approx 100$.

The plasma flow across the perpendicular magnetic field introduces a convective electric field in the y-direction with the magnitude $E_{c}=\left|\left(v_{c}, 0,0\right) \times\left(B_{x}, 0, B_{z}\right)\right| \approx 95 \mathrm{~V} / \mathrm{m}$. We initially set here $\boldsymbol{E}=0$ in the simulation box, while the sim-
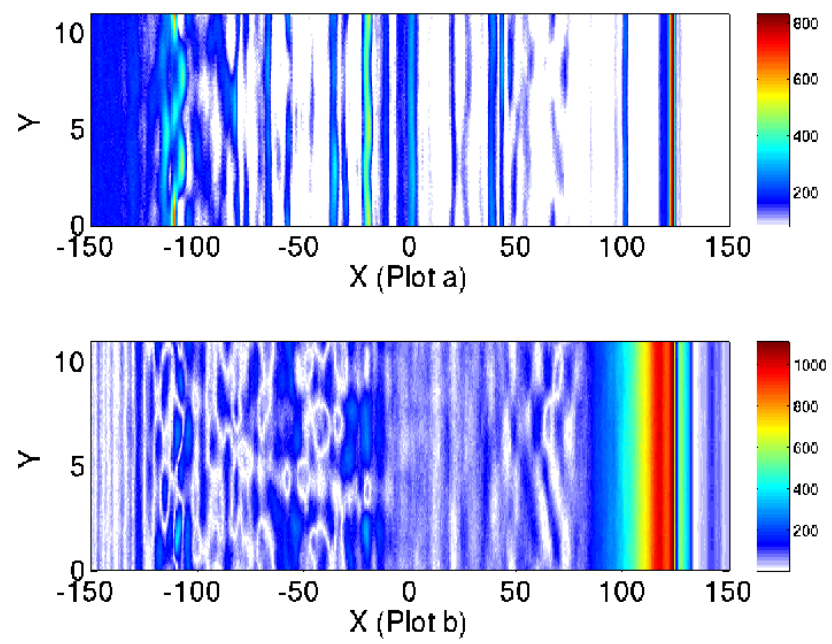

Fig. 7. The electric fields in $\mathrm{V} / \mathrm{m}$ in the 2-D simulation at $t=t_{2}$ : (a) shows the flow-aligned electric field component $E_{x}$ and (b) shows $E_{y}$, the second electric field component in the simulation plane.

ulation study by Dieckmann et al. (2008) introduced it. The $\boldsymbol{E}=0$ in the simulation box at $t=0$ implies that a wave electric field cancels the convective electric field at this time. This wave oscillates for $t>0$. The plasma oscillations do not introduce any detectable difference between the key simulation results discussed here and by Dieckmann et al. (2008). This is an important finding in itself, because colliding energetic plasma clouds in astrophysical jets are likely to carry with them strong plasma waves and it has to be demonstrated that the phase space structure is robust against such oscillations.

The 1-D simulation box length $L=1.58 \times 10^{4} \lambda_{s}$ is resolved by $1.8 \times 10^{5}$ cells. The $2-\mathrm{D}$ box has the extent $L_{x}=714 \lambda_{s}$ $\left(1.6 \times 10^{4}\right.$ cells) and $L_{y}=11 \lambda_{s}$ (200 cells) along $x$ and $y$, respectively. The boundary conditions are periodic. The 1-D (2-D) simulation time is $t_{1}=4370\left(t_{2}=194\right)$. The 2-D simulation represents each plasma species by 64 particles per cell (PPC). The 1-D simulation resolves each species of the dense (tenuous) cloud by 576 (256) PPC.

The electric field in the 2-D simulation plane at $t=t_{2}$ in Fig. 7 reveals a planar structure at $x \approx 120$, the leading edge of the dense cloud. Its amplitude far exceeds $E_{c}$, which is why the convective electric field does not influence the simulation results. A similar $E_{z}$ distribution is not shown. The filamentation instability leads to the structure formation on a scale of $\lambda_{s}$ along $y$ (Frederiksen et al., 2004; Dieckmann et al., 2008), and it is here suppressed by $B_{x}$.

We exploit the planarity of the cloud front in Fig. 7 and resort to the 1-D simulation with its better statistical plasma representation. The current system sampled at $t=t_{2}$ in the 1-D simulation is displayed in Fig. 8.

The electrons of the tenuous cloud are deflected by the oblique magnetic field that is frozen into the dense downstream plasma. Since the massive upstream ions are not sig- 

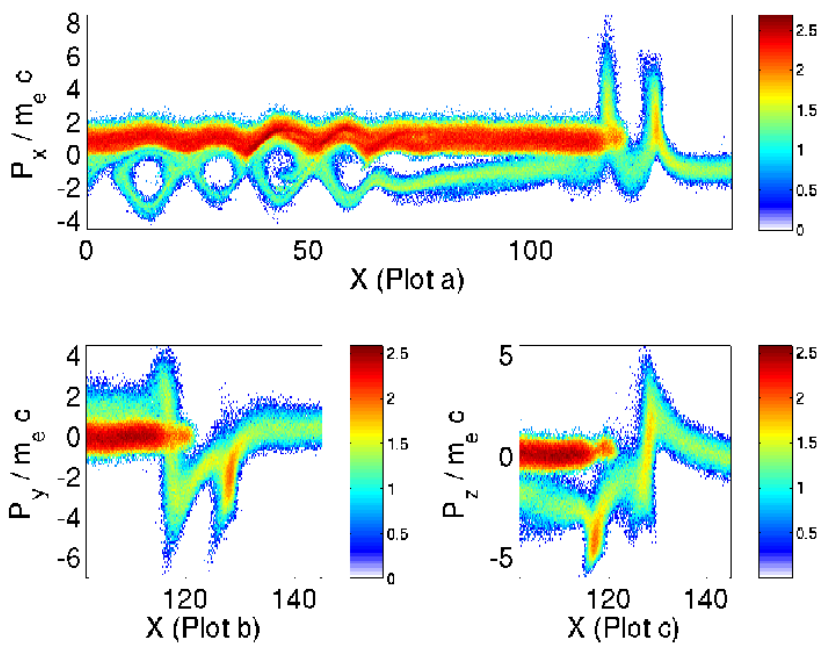

Fig. 8. The 10-logarithms of the electron distributions in the 1-D simulation at $t=t_{2}$ : The distribution in the $x, p_{x}$ plane is shown in (a). (b) and (c) display the distributions in the $x, p_{y}$ and $x, p_{z}$ planes.
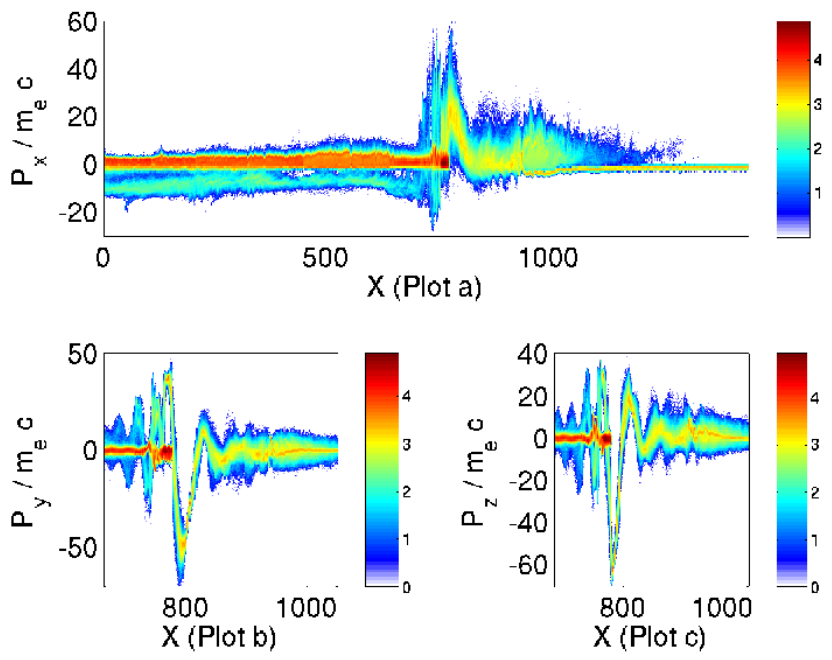

Fig. 9. The 10-logarithms of the electron distributions in the 1-D simulation at $t=1550$ : (a) shows the distribution $x, p_{x}$. The distributions in $x, p_{y}$ and $x, p_{z}$ are exhibited in (b) and (c).

nificantly deflected, a current and thus the electric field is building up at the leading edge of the dense cloud. This field is equivalent to the planar field at $x \approx 120$ in Fig. 7. Figure 8a shows the electron phase space holes farther downstream.

The energy dissipation by the electric fields eventually modulates the electrons of the dense cloud, giving rise to larger electric fields. Figure 9 consequently demonstrates that the electrons are accelerated to $\Gamma$ factors of tens and follow a corkscrew orbit. The relativistic mass of the electrons is not small compared to $m_{i}=400 m_{e}$ at $t=1550$.
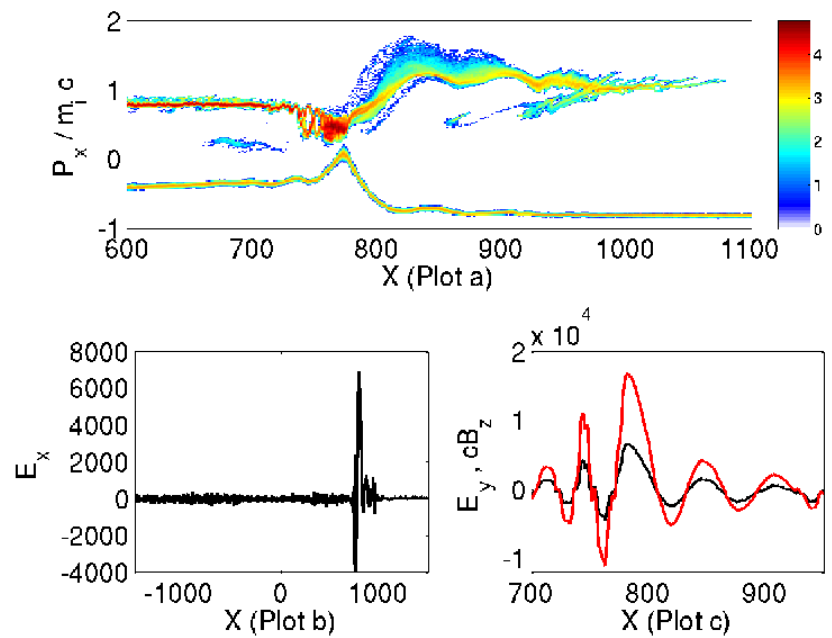

Fig. 10. (a) shows the ion distribution in $x, p_{x}$ space. The color is the 10-logarithm of the number of CPs. (b) shows the electrostatic $E_{x}$ field and (c) shows $E_{y}$ and $c B_{z}$ (red). The time is $t=1550$.

Figure 10a shows the ion response to the strong fields.

The spatial interval with the strongest particle acceleration at $x \approx 780$ is well behind the expected cloud front $v_{c} t / \lambda_{s} \approx 980$ at $t=1550$. Some ions have been accelerated beyond $v_{c}$ by the electric field at the cloud front.

The wave modes at $x \approx 800$ that grow only close to the leading edge of the dense plasma cloud, as Fig. 10b exemplifies for the $E_{x}$ field component, could have originated from an oblique instability. This instability does not grow if the plasma flows parallel to $\boldsymbol{B}_{0}$ (Bret et al., 2006; Hededal and Nishikawa, 2005; Dieckmann et al., 2006b). A weak perpendicular component of $\boldsymbol{B}_{0}$ and non-thermal plasma distributions in the collision area may thus be required. The wave growth at $x<0$ is suppressed or delayed. This will introduce an asymmetric plasma dynamics in the intervals separated by $x=0$.

The wave is circularly polarized, which explains the electron corkscrew orbits in Fig. 9. The peak value of $B_{z}$ in Fig. 10c exceeds the initial background magnetic field $B_{0}=\left(B_{x}^{2}+B_{z}^{2}\right)^{1 / 2}$ with $c B_{0} \approx 1500 \mathrm{~V} / \mathrm{m}$, and the wave in Fig. $10 \mathrm{c}$ is nonlinear. Their mixed polarity allows the waves to interact with the plasma through their electrostatic component. The wave amplitude is sustained, because the wave is driven by the dense cloud that compensates damping losses. The damping may explain why $E_{y}$ and $B_{z}$ are in phase in Fig. 10(c).

At $t=t_{1}$, the ion distribution in Fig. 11a has developed into a shock.

The foreshock in the interval $2000<x<3200$ is formed by incoming and shock-reflected ions. The electron transport with the shock-reflected ions across $\boldsymbol{B}_{0}$ in Fig. $11 \mathrm{~b}$ ensures the plasma quasi-neutrality. The two ion beams at $x<500$ 
result from the delayed shock formation. At $x \approx 1800$, the $p_{x}$ values of the electrons are comparable to those of the ions multiplied by $m_{i} / m_{e}$. A Lorentz transformation of the electron velocity into the upstream frame, which moves with $v=-v_{c}$ relative to the box frame, yields a peak electron $\Gamma \approx 700$ for our $v_{b}=0.9 c$.

The magnetic energy density in the downstream region equals the kinetic energy density of the electrons and ions (Dieckmann et al., 2008). The magnetic field component perpendicular to $\boldsymbol{v}_{b}$ is amplified by the shock, while the component of $\boldsymbol{B}_{0}$ along $\boldsymbol{v}_{b}$ does not change. Note, however, that the component of $\boldsymbol{B}_{0}$ along $\boldsymbol{x}$ cannot change in the 1-D simulation by $\nabla \cdot \boldsymbol{B}=0$. The 2-D simulations in (Dieckmann et al., 2008) suggest, however, that the flow-aligned magnetic field does not change very much also in multi-dimensional simulations, at least during the early stage of the instability. The shock develops into a quasi-perpendicular shock, which is a good ion reflector. The shock-reflected ion beam can outrun the shock, because $\boldsymbol{B}_{0}$ ahead of the shock is almost aligned with $\boldsymbol{v}_{b}$.

\section{Conclusions}

Particle-in-cell simulations are being used as computer experiments to better understand the plasma dynamics in energetic astrophysical plasmas. In particular the relativistic plasma jets of compact objects, such as neutron stars or black holes, or forming compact objects are of interest. Examples are the jets of microquasars, which are driven by stellarmass black holes that extract mass from their companion star (Fender and Belloni, 2004; Lindfors et al., 2007). The jets of active galactic nuclei (Harris and Krawczynski, 2002; Brunetti et al., 2002) are driven by the supermassive black holes that reside in the centres of galaxies (Melia and Falcke, 2001). Long gamma ray bursts are associated with the ultrarelativistic jet that is ejected, when a massive star implodes at the end of its lifetime (Piran, 2004).

The jets are powerful sources of electromagnetic emissions, some due to synchrotron radiation. Highly relativistic electrons and strong magnetic fields are required for the generation of synchrotron radiation. Both are thought to be produced by the mildly relativistic internal shocks that move through these jets (Waxman, 2006). How the magnetic fields and the relativistic electrons are generated is not yet fully understood. The plasma dynamics in the shock transition layer involves plasma processes on a kinetic (micro-)scale, while the shock dimensions are macroscopic. The simultaneous treatment of both scales is difficult (Siewert and Fahr, 2007).

The PIC simulations are computationally expensive and the accessible spatio-temporal scales are thus limited. Threedimensional PIC simulations of relativistic shocks are not yet feasible unless the ion-to-electron mass ratio is reduced. Such a reduction can, however, change the wavenumber spectrum of the unstable wave modes (Bret and Dieckmann,
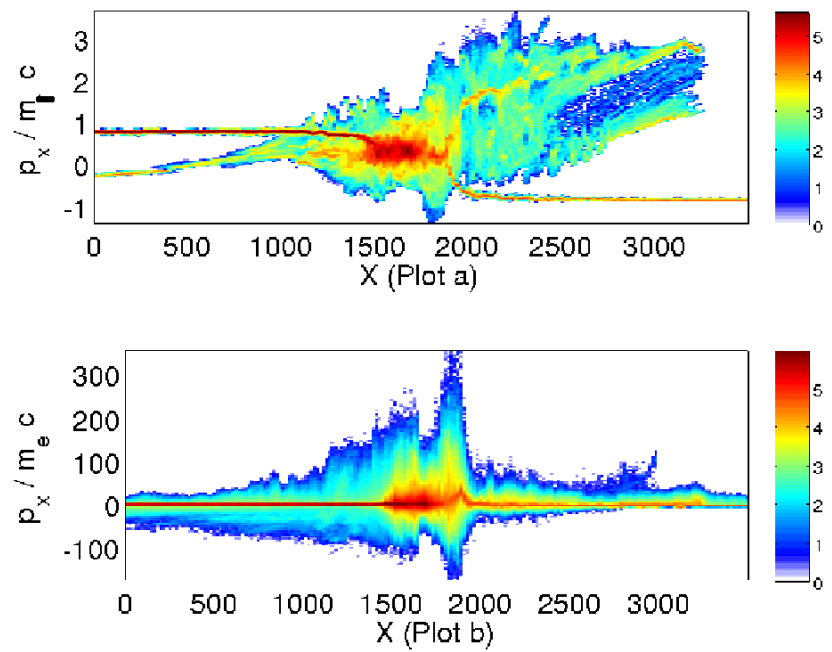

Fig. 11. The shock: (a) shows the ion distribution in $x, p_{x}$, while (b) shows the corresponding electron distribution. The color is the 10-logarithm of the number of CPs.

2008 ) and the correct instability may not develop. An expansion of the spatio-temporal scales by going to a 2-D system is also not completely realistic, if the nonlinear development of the instabilities is not resolved. The current channels, which develop out of the filamentation instability, can merge in 3-D simulations of colliding plasmas, but not in 2-D ones (Morse and Nielson, 1971; Dieckmann et al., 2007; Rowlands et al., 2007), where the flux tubes reach a steady state after some initial mergers.

Nevertheless, the PIC simulations can reveal the nonlinear evolution of instabilities in relativistic plasma and they can give insight into the formation and development of relativistic shocks. Selecting appropriate initial conditions and simulation geometries can isolate individual instabilities, which can then be examined separately. Aligning a 1-D simulation box with the flow velocity vector of a plasma beam that moves through an unmagnetized bulk plasma will isolate electrostatic instabilities like the Buneman instability (Buneman, 1959) or the two-stream instability (Morse and Nielson, 1969; Lapuerta and Ahedo, 2002). Upper-hybrid modes and electron cyclotron (Bernstein) modes are grown by particle beams in the presence of a perpendicular magnetic field. If the beam speed is mildly relativistic, the upper-hybrid modes are partially electromagnetic and they can amplify a background magnetic field (Dieckmann et al., 2008). The filamentation instability can be examined, if a $1 \mathrm{D}$ or a $2 \mathrm{D}$ simulation box is oriented orthogonally to the flow velocity vectors of two counter-propagating beams (Morse and Nielson, 1971). Individual mixed modes can be selected by tilting the 1-D simulation box relative to the flow velocity vector of a plasma beam (Dieckmann et al., 2006). 
The Sect. 3 has revised simulation studies that focused on either the generation of magnetic fields or on the particle acceleration. The growth of magnetic fields has either been due to the filamentation instability or by the Weibel instability. The filamentation instability is driven by beams of charged particles, whereas a thermally anisotropic plasma relaxes through the Weibel instability (Tzoufras et al., 2007). Both instabilities result in the aperiodic growth of purely electromagnetic waves. These waves saturate by the formation of current filaments. The saturation mechanism is either the growth of electrostatic waves by the magnetic pressure gradients (Rowlands et al., 2007; Stockem et al., 2008) that displaces electrons, or by the magnetic trapping mechanism (Davidson et al., 1972). After the instabilities saturate, the current filaments merge (Bogdan and Lerche, 1985). The typical filament size increases linearly (Dieckmann et al., 2007) or close to linearly (Medvedev et al., 2005) with the time, if they develop out of counter-propagating equal electron beams.

Magnetic fields can also be grown by the mixed modes. These waves have a mixed electrostatic and electromagnetic polarity (Bludman et al., 1960; Bret et al., 2004). While they saturate through the formation of islands of trapped electrons in phase space (Dieckmann et al., 2006), their magnetic field component is not negligible and they may yield magnetic field distributions that are similar to those of the filamentation instability.

It is possible though that the final electron distribution can be used to determine whether relativistically colliding plasmas thermalized through the filamentation instability or through the mixed mode instability. The first one results in electron momentum distributions that decrease faster than exponential (Nishikawa et al., 2006; Dieckmann et al., 2007), while the mixed modes yield electron momentum distributions that resemble a power-law (Hededal et al., 2004; Dieckmann et al., 2006). The relativistic Jüttner-Synge distribution that decreases faster than exponential and power-law distributions can both constitute a relativistic equilibrium plasma (Kaniadakis, 2005; Treumann and Jaroschek, 2008). Future studies have to address this issue in more detail by comparing systems of colliding plasmas, in which only single relevant parameters are varied, i.e. the beam density ratio.

The formation of phase space holes in the electron and ion distribution (Roberts and Berk, 1967; Luque and Schamel, 2005; Eliasson and Shukla, 2006) is also the saturation mechanism of the purely electrostatic two-stream and Buneman instabilities. We have discussed a Buneman-type instability (BTI) due to the interaction of a dense relativistic ion beam with the bulk electrons based on the simulations by Dieckmann et al. (2004), Dieckmann et al. (2006a) and Dieckmann et al. (2007). The initial saturation of the BTI can transfer only a minor fraction of the ion beam energy to the wave and to the electrons. The peak energy of the electrons is thus limited. The PIC simulations demonstrate, however, that the interaction of non-uniformly distributed and highly relativistic electrons with the ion beam can trigger a secondary electrostatic instability. This instability is capable of thermalizing the ion beam. A huge ion phase space hole forms that can accelerate the electrons to ultrarelativistic speeds. An energy equilibrium between electrons and ions is established. The comparison of the simulations by Dieckmann et al. (2004) and by Dieckmann et al. (2007) indicates that dense beams of ions are necessary. Future work has to assess the lowest beam density, for which such instabilities develop. It has to be tested if and when this instability can develop in more realistic 2-D PIC simulations. The electron acceleration by the upper-hybrid modes is, for example, decreased drastically as we go from a 1D to a 2-D system (Dieckmann et al., 2008)

Systems of colliding plasma clouds accelerate electrons to ultrarelativistic speeds and amplify magnetic fields to extreme amplitudes (Bessho and Ohsawa, 1999, 2000; Dieckmann et al., 2008), as we have discussed in the Sect. 4. We have considered plasma clouds with inequal densities, which collide at mildly relativistic speeds. A magnetic field $\boldsymbol{v}_{b}$ has been oriented obliquely to the flow velocity vector $\boldsymbol{v}_{b}$. The component of $\boldsymbol{B}_{0}$ parallel to $\boldsymbol{v}_{b}$ has suppressed the growth of the filamentation modes and of the mixed modes. The PIC simulation has demonstrated that the initially weak magnetic field component perpendicular to $\boldsymbol{v}_{b}$ is amplified by the collision. It grows to a strength that far exceeds that of the flowaligned magnetic field component. It establishes an equipartition between the ion and electron kinetic energy and the magnetic energy downstream and it reflects a large fraction of the incoming upstream ions. The shock-reflected ion beam can escape the shock because the upstream magnetic field is still almost aligned with the shock normal. The moderately relativistic ion beam can be thermalized through BTIs, which would heat up the electrons. The electron pre-acceleration ahead of the shock could inject some electrons into a Fermiacceleration across the mildly relativistic oblique shock, the onset of which may have already be found in PIC simulations (Spitkovsky, 2008b). The electrons could reach cosmic ray energies through this process (Meli and Biermann, 2006; Drury, 1983).

Particle-in-cell simulations are thus an increasingly important tool to address particle acceleration and magnetic field generation in astrophysical environments. Global shock simulations indicate that shocks can accelerate electrons to ultrarelativistic speeds through plasma instabilities and that magnetic fields can reach energies comparable to those of the electrons and ions. These processes are non-thermal and involve a wide range of phase space structures, of which we have here discussed current filaments, electron and ion phase space holes, and corkscrew distributions. The filamentation instability is usually associated only with the generation of magnetic fields. PIC simulations show that it may also be a powerful electron accelerator if the flow speeds are ultrarelativistic (Spitkovsky, 2008a). The electrostatic ion holes are good electron accelerators and they may also drive the Weibel instability through the thermal anisotropy gen- 
erated by their monodirectional electron acceleration. The corkscrew distributions can simultaneously accelerate electrons and produce equi-partition magnetic fields and they develop in mildly relativistic flows.

Several plasma processes can thus result in the production of ultrarelativistic electrons and strong magnetic fields. The simultaneous presence of both components is required to explain the synchrotron emissions of astrophysical jets and PIC simulations are now starting to demonstrate that even mildly relativistic plasma instabilities and realistic initial conditions can result in such a scenario.

Acknowledgements. Mark Dieckmann thanks Vetenskapsrådet for financially supporting this work. The Irish centre for high-end computing (ICHEC) and the Swedish high-performance computer center north (HPC2N) have provided the computers and support.

Edited by: W. M. Macek

Reviewed by: three anonymous referees

\section{References}

Balbus, S. A. and Hawley, J. F.: Instability, turbulence, and enhanced transport in accretion disks, Rev. Mod. Phys., 70, 1-53, 1998.

Bessho, N. and Ohsawa, Y.: Energies of ultrarelativistic electrons produced by an oblique shock wave, Phys. Plasmas, 7, 40044012, 2000.

Bessho, N. and Ohsawa, Y.: Electron acceleration to ultrarelativistic energies in a collisionless oblique shock wave, Phys. Plasmas, 3076-3085, 1999.

Birdsall, C. K. and Langdon, A. B.: Plasma physics via computer simulation, IOP Publishing, Bristol, UK, 1991.

Bludman, S. A., Watson, K. M., and Rosenbluth, M. N.: Statistical mechanics of relativistic streams. II, Phys. Fluids, 3, 747-757, 1960.

Bogdan, T. J. and Lerche, I.: Dynamical evolution of large-scale, two-dimensional, fibril magnetic-fields, Astrophys. J., 296, 719738, 1985.

Bret, A. and Dieckmann, M. E.: Ions motion effects on the full unstable spectrum in relativistic electron beam plasma interaction, Phys. Plasmas, 15, 012104, doi:10.1063/1.2828607, 2008.

Bret, A., Dieckmann, M. E., and Deutsch, C.: Oblique electromagnetic instabilities for a hot relativistic beam interacting with a hot and magnetized plasma, Phys. Plasmas, 13, 082109, doi:10.1063/1.2335414, 2006.

Bret, A., Firpo, M. C., and Deutsch, C.: Collective electromagnetic modes for beam-plasma interaction in the whole k space, Phys. Rev. E., 70, 046401, doi:10.1103/PhysRevE.70.046401, 2004.

Bret, A., Gremillet, L., and Bellido, J. C.: How really transverse is the filamentation instability, Phys. Plasmas, 14, 032103, doi:10.1063/1.2710810, 2007.

Brunetti, G., Bondi, M., Comastri, A., and Setti, G.: Chandra discovery of extended non-thermal emission in 3C 207 and the spectrum of the relativistic electrons, Astron. Astrophys., 381, 795$809,2002$.
Buneman, O.: Dissipation of currents in ionized media, Phys. Rev., 115, 503-517, 1959.

Böttcher, M.: Astrophysical jets of blazars and microquasars, Astrophys. Space Sci., 307, 69-75, 2007.

Califano, F., Cecchi, T. and Chiuderi, C.: Nonlinear kinetic regime of the Weibel instability in an electron-ion plasma, Phys. Plasmas, 9, 451-457, 2002.

Califano, F., Del Sarto, D., and Pegoraro, F.: Three-dimensional magnetic structures generated by the development of the filamentation (Weibel) instability in the relativistic regime, Phys. Rev. Lett., 96, 105008, doi:10.1103/PhysRevLett.96.105008, 2006.

Chang, P., Spitkovsky, A., and Arons, J.: Long-term evolution of magnetic turbulence in relativistic collisionless shocks: Electronpositron plasmas, Astrophys. J., 674, 378-387, 2008.

Chapman, S. C., Lee, R. E., and Dendy, R. O.: Perpendicular shock reformation and ion acceleration, Space Sci. Rev., 121, 5-19, 2005.

Das, T. K., Rao, A. R., and Vadawale, S. V.: Quasi-periodic oscillation frequencies and mass-outflow rates in black hole powered Galactic microquasars, Mon. Not. R. Astron. Soc., 343, 443-455 2003.

Davidson, R. C., Hammer, D. A., Haber, I., and Wagner, C. E.: Nonlinear development of electromagnetic instabilities in anisotropic plasmas, Phys. Fluids, 15, 317-333, 1972.

Dawson, J. M.: Particle simulation of plasmas, Rev. Mod. Phys., 55, 403-447, 1983.

Dieckmann, M. E., Bret, A., and Shukla, P. K.: Comparing electrostatic instabilities driven by mildly and highly relativistic proton beams, Plasma Phys. Controll. Fusion, 49, 1989-2004, 2007.

Dieckmann, M. E., Bret, A., and Shukla, P. K.: Electron surfing acceleration by mildly relativistic beams: wave magnetic field effects, New. J. Phys., 10, 013029, doi:10.1088/13672630/10/1/013029, 2008.

Dieckmann, M. E., Eliasson, B., and Shukla, P. K.: Electron acceleration to energies beyond $\mathrm{GeV}$ by a relativistic ion beam instability, Phys. Rev. E., 70, 036401, doi:10.1103/PhysRevE.70.036401, 2004.

Dieckmann, M. E., Frederiksen, J. T., Bret, A., and Shukla, P. K.: Evolution of the fastest-growing relativistic mixed mode instability driven by a tenuous plasma beam in one and two dimensions, Phys. Plasmas, 13, 112110, doi:10.1063/1.2390687, 2006.

Dieckmann, M. E., Lerche, I., Shukla, P. K., and Drury, L. O. C.: Aspects of self-similar current distributions resulting from the plasma filamentation instability, New J. Phys., 9, 10, 2007.

Dieckmann, M. E., Shukla, P. K., and Drury, L. O. C.: Particle-incell simulation studies of the non-linear evolution of ultrarelativistic two-stream instabilities, Mon. Not. R. Astron. Soc., 367, 1072-1082, 2006.

Dieckmann, M. E., Shukla, P. K., and Drury, L. O. C.: The formation of a relativistic partially electromagnetic planar plasma shock, Astrophys. J., 675, 586-597, 2008.

Dieckmann, M. E., Shukla, P. K., and Eliasson, B.: Formation of electrostatic structures by wakefield acceleration in ultrarelativistic plasma flows: Electron acceleration to cosmic ray energies, Phys. Plasmas, 13, 062905, doi:10.1063/1.2212393, 2006a.

Dieckmann, M. E., Shukla, P. K., and Eliasson, B.: Particle-incell simulations of plasma slabs colliding at a mildly relativistic speed, New J. Phys., 8, 225, doi:10.1088/1367-2630/8/10/225, $2006 b$. 
Drury, L. O. C.: An introduction to the theory of diffusive shock acceleration of energetic particles in tenuous plasmas, Rep. Prog. Phys., 46, 973-1027, 1983.

Dupree, T. H.: Kinetic theory of plasma and the electromagnetic field, Phys. Fluids, 6, 1714-1729, 1963.

Eastwood, J. P.: The virtual particle electromagnetic particle-mesh method, Comput. Phys. Comm., 64, 252-266, 1991.

Eastwood, J. P., Lucek, E. A., Mazelle, C., Meziane, K., Narita, Y., Pickett, J., and Treumann, R. A.: The foreshock, Space Sci. Rev., 118, 41-94, 2005.

Eliasson, B. and Shukla, P. K.: Theory of relativistic electron holes in hot plasmas, Phys. Lett. A, 340, 237-242, 2005.

Eliasson, B. and Shukla, P. K.: Formation and dynamics of coherent structures involving phase-space vortices in plasmas, Phys. Rep., 422, 225-290, 2006.

Fender, R. and Belloni, T.: GRS 1915+105 and the disc-jet coupling in accreting black hole systems, Ann. Rev. Astron. Astrophys., 42, 317-364, 2004.

Fiore, M., Silva, L. O., Ren, C., Tzoufras, M. A., and Mori, W. B.: Baryon loading and the Weibel instability in gamma-ray bursts, Mon. Not. R. Astron. Soc., 372, 1851-1855, 2006.

Fleishman, G. D. and Toptygin, I. N.: Diffusive radiation in Langmuir turbulence produced by jet shocks, Mon. Not. R. Astron. Soc., 381, 1473-1481, 2007.

Fonseca, R. A., Silva, L. O., Tonge, J. W., Mori, W. B., and Dawson, J. M.: Three-dimensional Weibel instability in astrophysical scenarios, Phys. Plasmas, 10, 1979-1984, 2003.

Forslund, D. W. and Freidberg, J. P.: Theory of laminar collisionless shocks, Phys. Rev. Lett., 27, 1189-1192, 1971.

Forslund, D. W. and Shonk, C. R.: Formation and structure of electrostatic collisionless shocks, Phys. Rev. Lett., 25, 1699-1702, 1970.

Fox, D. B. and Meszaros, P.: GRB fireball physics: prompt and early emission, New J. Phys., 8, 199, doi:10.1088/13672630/8/9/199, 2006.

Frederiksen, J. T., Hededal, C. B., Haugbolle, T., and Nordlund, A.: Magnetic field generation in collisionless shocks: Pattern growth and transport, Astrophys. J., 608, L13-L16, doi:10.1086/421262, 2004.

Gruzinov, A. and Waxman, E.: Gamma-ray burst afterglow: Polarization and analytic light curves, Astrophys. J., 511, 852-861, 1999.

Harris, D. E. and Krawczynski, H.: X-ray emission processes in radio jets, Astrophys. J., 565, 244-255, 2002.

Harris, D. E. and Krawczynski, H.: X-ray emission from extragalactic jets, Ann. Rev. Astron. Astrophys., 44, 463-506, 2006.

Hededal, C. B., Haugbolle, T., Frederiksen, J. T., and Nordlund, A.: Non-Fermi power-law acceleration in astrophysical plasma shocks, Astrophys. J., 617, L107-L110, doi:10.1086/427387, 2004.

Hededal, C. B. and Nishikawa, K. I.: The influence of an ambient magnetic field on relativistic collisionless plasma shocks, Astrophys. J., 623, L89-L92, doi:10.1086/430253, 2005.

Hellinger, P., Travnicek, P., Lembege, B., and Savoini, P.: Emission of nonlinear whistler waves at the front of perpendicular supercritical shocks: Hybrid versus full particle simulations, Geophys. Res. Lett., 34, L14109, doi:10.1029/2007GL030239, 2007.
Hirose, A., Ishihara, O., and Langdon, A. B.: Non-linear evolution of the Buneman instability. II. Ion dynamics, Phys. Fluids, 25, 610-616, 1982.

Hockney, R. W. and Eastwood, J. W.: Computer Simulation Using Particles, Taylor and Francis, Bristol, PA, USA, 1989.

Honda, M.: Eigenmodes and growth rates of relativistic current filamentation instability in a collisional plasma, Phys. Rev. E., 69, 016401, doi:10.1103/PhysRevE.69.016401, 2004.

Honda, M., Meyer-ter-Vehn, J., and Pukhov, A.: Collective stopping and ion heating in relativistic-electron-beam transport for fast ignition, Phys. Rev. Lett., 85, 2128-2131, 2000.

Hoshino, M. and Shimada, N.: Nonthermal electrons at high Mach number shocks: Electron shock surfing acceleration, Astrophys. J., 572, 880-887, 2002.

Ishihara, O., Hirose, A., and Langdon, A. B.: Non-linear saturation of the Buneman instability, Phys. Rev. Lett., 44, 1404-1407, 1980.

Ishihara, O., Hirose, A., and Langdon, A. B.: Non-linear evolution of the Buneman instability, Phys. Fluids, 24, 452-464, 1981.

Jaroschek, C. H., Lesch, H., and Treumann, R. A.: Self-consistent diffusive lifetimes of Weibel magnetic fields in gamma-ray bursts, Astrophys. J., 616, 1065-1071, 2004.

Kato, T. N.: Relativistic collisionless shocks in unmagnetized electron-positron plasmas, Astrophys. J., 668, 974-979, 2007.

Kaniadakis, G.: Statistical mechanics in the context of special relativity. II., Phys. Rev. E., 72, 036108, doi:10.1103/PhysRevE.72.036108, 2005.

Kazimura, Y., Sakai, J. I., Neubert, T. and Bulanov, S. V.: Generation of a Small-Scale Quasi-Static Magnetic Field and Fast Particles during the Collision of Electron-Positron Plasma Clouds, Astrophys. J. 498, L183-L186, doi:10.1086/311316, 1998.

Kuramitsu, Y. and Krasnoselskikh, V.: Gyroresonant surfing acceleration, Phys. Rev. Lett., 94, 031102 , doi:10.1103/PhysRevLett.94.031102, 2005.

Lancellotti, C. and Dorning, J. J.: Time-asymptotic wave propagation in collisionless plasmas, Phys. Rev. E., 68, 026406, doi:10.1103/PhysRevE.68.026406, 2003.

Langdon, A. B., Arons, J., and Max, C. E.: Structure of relativistic magnetosonic shocks in electron-positron plasmas, Phys. Rev. Lett., 61, 779-782, doi:10.1103/PhysRevLett.61.779, 1988.

Lapenta, G., Markidis, S., Marocchino, A., and Kaniadakis, G.: Relaxation of relativistic plasmas under the effect of wave-particle interactions, Astrophys. J., 666, 949-954, 2007.

Lapuerta, V. and Ahedo, E.: General parametric analysis of the linear two-stream instability, Phys. Plasmas, 9, 1513-1519, 2002.

Lazar, M., Schlickeiser, R., and Shukla, P. K.: Cumulative effect of the filamentation and Weibel instabilities in counterstreaming thermal plasmas, Phys. Plasmas, 13, 102107, doi:10.1063/1.2357047, 2006.

Lembege, B. and Dawson, J. M.: Self-consistent study of a perpendicular collisionless and nonresistive shock, Phys. Fluids, 30 1767-1788, 1987.

Lembege, B. and Dawson, J. M.: Formation of double-layers within an oblique collisionless shock, Phys. Rev. Lett., 62, 2683-2686, 1989.

Lembege, B., Giacalone, J., Scholer, M., Hada, T., Hoshino, M., Krasnoselskikh, V., Kucharek, H., Savoini, P., and Terasawa, T.: Selected problems in collisionless-shock physics, Space Sci. Rev., 110, 161-226, 2004. 
Levinson, A.: Electron injection in collisionless shocks, Astrophys. J., 401, 73-80, 1992.

Lindfors, E. J., Türler, M., Hannikainen, D. C., Pooley, G., Tammi, J., Trushkin, S. A., and Valtaoja, E.: Synchrotron flaring behaviour of Cygnus X-3 during the February-March 1994 and September 2001 outbursts, Astron. Astrophys., 473, 923-929, 2007.

Luque, A. and Schamel, H.: Electrostatic trapping as a key to the dynamics of plasmas, fluids and other collective systems, Phys. Rep., 415, 261-359, 2005.

Matsukiyo, S. and Scholer, M.: On reformation of quasi-parallel collisionless shocks, Adv. Space Res., 38, 57-63, 2006.

McClements, K. G., Dendy, R. O., Bingham, R., Kirk, J. G., and Drury, L. O.: Acceleration of cosmic ray electrons by ion-excited waves at quasi-perpendicular shocks, Mon. Not. R. Astron. Soc., 291, 241-249, 1997.

Medvedev, M. V. and Loeb, A.: Generation of magnetic fields in the relativistic shock of gamma-ray burst sources, Astrophys. J., 526, 697-706, 1999.

Medvedev, M. V., Fiore, M., Fonseca, R. A., Silva, L. O., and Mori, W. B.: Long-time evolution of magnetic fields in relativistic gamma-ray burst shocks, Astrophys. J., 618, L75-L78, doi:10.1086/427921, 2005.

Meli, A. and Biermann, P. L.: Cosmic rays X. The cosmic ray knee and beyond: diffusive acceleration at oblique shocks, Astron. Astrophys., 454, 687-694, 2006.

Melia, F. and Falcke, H.: The supermassive black hole at the galactic centre, Ann. Rev. Astron. Astrophys., 39, 309-352, 2001.

Molvig, K.: Filamentary instability of a relativistic electron beam, Phys. Rev. Lett., 35, 1504-1507, 1975.

Morse, R. L. and Nielson, C. W.: One-, Two-, and Threedimensional numerical simulations of two-beam plasmas, Phys. Rev. Lett., 23, 1087-1090, 1969.

Morse, R. L. and Nielson, C. W.: Numerical simulation of Weibel instability in one and 2 dimensions, Phys. Fluids, 14, 830-840, 1971.

Nishikawa, K. I., Hardee, P. E., Hededal, C. B., and Fishman, G. J.: Acceleration mechanics in relativistic shocks by the Weibel instability, Astrophys. J., 642, 1267-1274, 2006.

Pe'er, A., Ryde, F., Wijers, R. A. M. J., Meszaros, P., and Rees, M. J.: A new method of determining the initial size and Lorentz factor of gamma-ray burst fireballs using a thermal emission component, Astrophys. J., 664, L1-L4, doi:10.1086/520534, 2007.

Pe'er, A. and Zhang, B.: Synchrotron emission in small-scale magnetic fields as a possible explanation for prompt emission spectra of gamma-ray bursts, Astrophys. J., 653, 454-461, 2006.

Piran, T.: The physics of gamma-ray bursts, Rev. Mod. Phys., 76, 1143-1210, 2004.

Roberts, K. V. and Berk, H. L.: Nonlinear evolution of a 2-stream instability, Phys. Rev. Lett., 19, 297-300, 1967.

Romanov, D. V., Bychenkov, V. Y., Rozmus, W., Capjack, C. E., and Fedosejevs, R.: Self-organization of a plasma due to 3-D evolution of the Weibel instability, Phys. Rev. Lett., 93, 215004, doi:10.1103/PhysRevLett.93.215004, 2004.

Roth, I.: Planetary, solar and astrophysical relativistic electrons: Common energization mechanisms?, Planet. Space Sci., 55, 2319-2323, 2007.
Rowlands, G., Dieckmann, M. E., and Shukla, P. K.: The plasma filamentation instability in one dimension: nonlinear evolution, New J. Phys., 9, 247, doi:10.1088/1367-2630/9/8/247, 2007.

Schmitz, H., Chapman, S. C., and Dendy, R. O.: Electron preacceleration mechanisms in the foot region of High Alfvenic Mach number shocks, Astrophys. J., 579, 327-336, 2002.

Shakura, N. I. and Sunyaev, R. A.: Black holes in binary-systems - observational appearance, Astron. Astrophys., 24, 337-355, 1973.

Shimada, N. and Hoshino, M.: Electron heating and acceleration in the shock transition region: Background plasma parameter dependence, Phys. Plasmas, 11, 1840-1849, 2004.

Shukla, P. K.: Purely growing electromagnetic mode driven by iontemperature anisotropy in a collisional plasma, Phys. Lett. A., 370, 316-318, 2007.

Siewert, M. and Fahr, H. J.: Full Boltzmann-kinetical treatment of an ion plasma crossing an MHD shock: parallel and non-parallel cases, Astron. Astrophys., 471, 7-15, 2007.

Silva, L. O.: Physical problems (microphysics) in relativistic plasma flows, AIP Conf. Proc., 856, 109-128,2006.

Silva, L. O., Fonseca, R. A., Tonge, J. W., Dawson, J. M., Mori, W. B., and Medvedev, M. V.: Interpenetrating plasma shells: Near-equipartition magnetic field generation and nonthermal particle acceleration, Astrophys. J., 596, L121-L124, doi:10.1086/379156, 2003.

Sorasio, G., Marti, M., Fonseca, R., and Silva, L. O.: Very high Mach-number electrostatic shocks in collisionless plasmas, Phys. Rev. Lett., 96, 045005, doi:10.1103/PhysRevLett.96.045005, 2006.

Spitkovsky, A.: On the structure of relativistic collisionless shocks in electron-ion plasmas, Astrophys. J., 673, L39-L42, doi:10.1086/527374, 2008a.

Spitkovsky, A.: Particle acceleration in relativistic collisionless shocks: Fermi process at last?, Astrophys. J., 682, L5-L8, doi:10.1086/590248, 2008b.

Stenzel, R. L., Urrutia, J. M., and Strohmaier, K. D.: Whistler modes with wave magnetic fields exceeding the ambient field, Phys. Rev. Lett., 96, 095004, doi:10.1103/PhysRevLett.96.095004, 2006.

Stockem, A., Dieckmann, M. E., and Schlickeiser, R.: Suppression of the filamentation instability by a flow-aligned magnetic field: testing the analytic threshold with PIC simulations, Plasma Phys. Controll. Fusion, 50, 025002, doi:10.1088/07413335/50/2/025002, 2008.

Tautz, R. C. and Schlickeiser, R.: Counterstreaming magnetized plasmas. II. Perpendicular wave propagation, Phys. Plasmas, 13, 062901, doi:10.1063/1.2207588, 2006.

Thode, L. E. and Sudan, R. N.: 2-stream instability heating of plasmas by relativistic electron beams, Phys. Rev. Lett., 30, 732-735, 1973.

Tokar, R. L. and Gary, S. P.: The whistler mode in a Vlasov-plasma, Phys. Fluids, 28, 1063-1068, 1985.

Treumann, R. A. and Jaroschek, C. H.: Gibbsian theory of power-law distributions, Phys. Rev. Lett., 100, 155005, doi:10.1103/PhysRevLett.100.155005, 2008.

Tzoufras, M., Ren, C., Tsung, F. S., Tonge, J. W., and Mori, W. B.: Stability of arbitrary electron velocity distribution functions to electromagnetic modes, Phys. Plasmas, 14, 062108, doi:10.1063/1.2740698, 2007. 
Tzoufras, M., Ren, C., Tsung, F. S., Tonge, J. W., Mori, W. B., Fiore, M., Fonseca, R. A., and Silva, L. O.: Space-charge effects in the current-filamentation or Weibel instability, Phys. Rev. Lett., 96, 105002, doi:10.1103/PhysRevLett.96.105002, 2006.

Waxman, E.: Gamma-ray bursts and collisionless shocks, Plasma Phys. Controll. Fusion, 48, B137-B151, doi:10.1088/07413335/48/12B/S14, 2006.

Weibel, E. S.: Spontaneously growing transverse waves in a plasma due to an anisotropic velocity distribution, Phys. Rev. Lett., 2, 83-84, 1959.
Yoon, P. H.: Relativistic Weibel instability, Phys. Plasmas, 14, 024504, doi:10.1063/1.2646285, 2007.

Yoon, P. H. and Davidson, R. C.: Exact analytical model of the classical Weibel instability in a relativistic anisotropic plasma, Phys. Rev. A, 35, 2718-2721, 1987. 